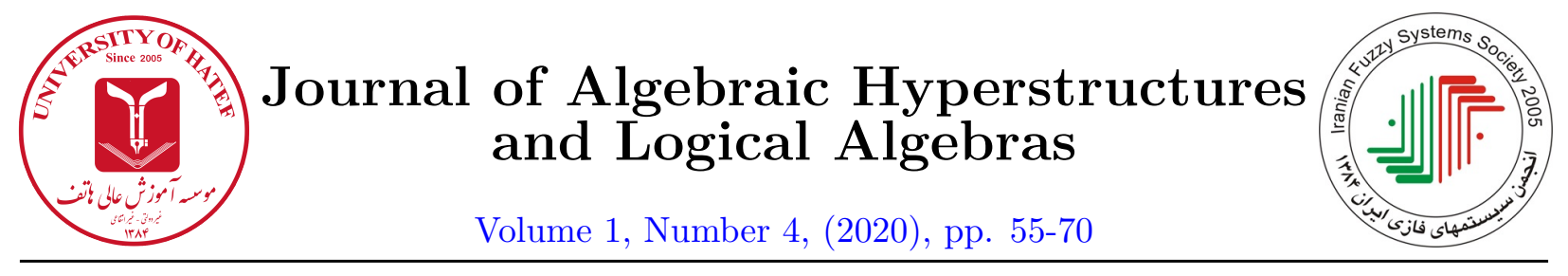

\title{
Characterization of ordered semihypergroups in terms of uni-soft bi-hyperideals
}

\author{
M. Farooq ${ }^{1}$, A. Khan ${ }^{2}$, R. Khan ${ }^{3}$ and M. Izhar ${ }^{4}$ \\ ${ }^{1,2}$ Department of Mathematics, Abdul Wali Khan University Mardan, 23200, Khyber Pakhtunkhwa \\ Pakistan \\ ${ }^{1}$ Govt. Higher Secondary School Mohib Banda Mardan, 23200, Khyber Pakhtunkhwa, Pakistan \\ ${ }^{3}$ Department of Mathematics, FATA University, Kohat, KP, Pakistan \\ ${ }^{4}$ Department of Mathematics, Govt. College Garhi Kapoora Mardan, 23200, Khyber Pakhtunkhwa Pakistan \\ farooq4math@gmail.com, azhar4set@yahoo.com, raeeskhan@fu.edu.pk, mizharmath@gmail.com
}

\begin{abstract}
In this paper, we introduce the concept of unionsoft (briefly, uni-soft) bi-hyperideal of an ordered semihypergroup. The notions of prime (strongly prime, semiprime, irreducible, and strongly irreducible) uni-soft bi-hyperideals in ordered semihypergroups are introduced and related properties are investigated. Numerous examples of these notions are given. The relationship between prime and strongly prime, irreducible and strongly irreducible uni-soft bi-hyperideals are considered and characterizations of these concepts are established. Regular and intra-regular ordered semihypergroups are characterized in terms of these notions.
\end{abstract}

\section{Article Information}

Corresponding Author:

M. Farooq;

Received: May 2020;

Revised: August 2020;

Accepted: August 2020,

Paper type: Original.

\section{Keywords:}

Uni-soft semi-hypergroup, prime (strongly prime), unisoft bi-hyperideal, semiprime uni-soft bi-hyperideal, irreducible (strongly irreducible) uni-soft bi-hyperideal, regular and intra-regular ordered semihypergroup.

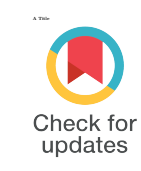

\section{Introduction}

The theory of soft sets is introduced by Molodtsov in [24]. The basic aim of this theory is to introduce a new tool to discuss uncertainty. In soft set theory, we have enough number of parameters

https://doi.org/10.29252/HATEF.JAHLA.1.4.4 
to deal with uncertainty. This quality makes it prominent among its predecessor theories such as probability theory, interval mathematics, fuzzy sets and rough sets. Hybrid soft set theories such as fuzzy soft sets and vague soft sets have been studied in [22, 28]. Theories of fuzzy sets and rough sets are quite different in their nature from soft sets, but there is a strong link among these three theories. During recent years, efforts have been made to establish links among these three theories [2, 10]. Initially, Maji et al. established the theoretical base for soft sets. They defined several operations on soft sets [21]. Later on it was felt that some of the operations defined for soft sets suffer many difficulties. In order to make these operations sensible, Ali et al. defined some new operations on soft sets [3]. Algebras, which appear as a natural consequence of these new operations have been studied in [4]. The theory of soft sets has emerged as a new tool to discuss algebraic structures. Study of soft algebraic structures was initiated by Aktas and Cagman. They studied soft groups in [1]. For application of soft sets in algebraic structures, see $[8,9,15,16,19,20,25,29]$.

In 1934 during the $8^{\text {th }}$ Congress of Scandinavian Mathematicians, F. Marty [23], introduced the concept of hyperstructures, analyzed its properties and applied them to groups, rational fractions and algebraic functions. After the pioneering work of F. Marty, algebraic hyperstructures have been developed by many researchers and widely studied from theoretical point of view and for their applications to many subjects of pure and applied mathematics. In a classical algebraic structures, the composition of two elements is an element, while in an algebraic hyperstructures, the composition of two elements is a set. Semihypergroups have been found useful for dealing with problems in different areas of algebraic hyperstructures. A short review of hyperstructures and some principal notions about hyperstructures and semihypergroups theory can be found in $[5,6,7,11,12,13,14,18,26,27]$.

Motivated by the study of Naz and Shabir [25], here in this paper, we apply uni-soft set theory to ordered semihypergroups and define the notion of uni-soft bi-hyperideals. Moreover, we define prime, strongly prime, semiprime, irreducible and strongly irreducible uni-soft bi-hyperideals of ordered semihypergrouprs. Different classes of ordered semihypergroups are characterized by the properties of uni-soft bi-hyperideals.

\section{Preliminaries}

A hypergroupoid is a nonempty set $S$ equipped with a hyperoperation $\circ$, that is a map $\circ: S \times S \longrightarrow$ $P^{*}(S)$, where $P^{*}(S)$ denotes the set of all nonempty subsets of $S$ (see [23]). We shall denote by $x \circ y$, the hyperproduct of elements $x, y$ of $S$. A hypergroupoid $(S, \circ)$ is called a semihypergroup if $(x \circ y) \circ z=x \circ(y \circ z)$ for all $x, y, z \in S$. Let $A, B$ be the nonempty subsets of $S$. Then the hyperproduct of $A$ and $B$ is defined as $A \circ B=\bigcup_{a \in A, b \in B} a \circ b$. We shall write $A \circ x$ instead of $A \circ\{x\}$ and $x \circ A$ for $\{x\} \circ A$.

Definition 2.1. [27] An algebraic hyperstructure $(S, \circ, \leq)$ is called an ordered semihypergroup (also called po-semihypergroup) if $(S, \circ)$ is a semihypergroup and $(S, \leq)$ is a partially ordered set such that the monotone condition holds as follows:

$a \leq b$ implies that $x \circ a \leq x \circ b$ and $a \circ x \leq b \circ x$ for all $x, a, b \in S$. If for every $a \in A$ there exists $b \in B$ such that $a \leq b$, then we can write as $A \preceq B$. If $A=\{a\}$, then we write $a \preceq B$ instead of $\{a\} \preceq B$.

Definition 2.2. [5] A nonempty subset $A$ of an ordered semihypergroup $(S, \circ, \leq)$ is called a subsemihypergroup of $S$ if for all $x, y \in A$, we have $x \circ y \subseteq A$. 
Equivalently: A nonempty subset $A$ of an ordered semihypergroup $(S, \circ, \leq)$ is called a subsemihypergroup of $S$ if $A \circ A \subseteq A$.

Definition 2.3. [27] Let $(S, \circ, \leq)$ be an ordered semihypergroup and $A$ be a nonempty subset of $S$. Then $A$ is called a left (right) hyperideal of $S$ if:

(1) $S \circ A \subseteq A((A \circ S) \subseteq A)$.

(2) If $a \in A$ and $b \in S$ such that $b \leq a$, then $b \in A$.

If $A$ is both a right hyperideal and a left hyperideal of $S$, then it is called a hyperideal of $S$.

For $A \subseteq S$, we denote $(A]=\{t \in S \mid t \leq h$ for some $h \in A\}$.

Definition 2.4. [27] A subsemihypergroup $A$ of an ordered semihypergroup $(S, \circ, \leq)$ is called a bi-hyperideal of $S$ if:

(1) $A \circ S \circ A \subseteq A$.

(2) If $a \in A$ and $b \in S$ such that $b \leq a$, then $b \in A$.

We denote by $B(a)$ the bi-hyperideal of $S$ generated by $a(a \in S)$. We have

$$
B(a)=(a \cup(a \circ a) \cup(a \circ S \circ a)] .
$$

Definition 2.5. [5] (1) An ordered semihypergroup $(S, \circ, \leq)$ is called regular if for each a $\in S$ there exists $x \in S$ such that $a \leq a \circ x \circ a$.

(2) An ordered semihypergroup $(S, \circ, \leq)$ is called intra-regular if for each a $\in S$ there exist $x, y \in S$ such that $a \leq x \circ a \circ a \circ y$.

Lemma 2.6. [12] Let $(S, \circ, \leq)$ be an ordered semihypergroup. Then the following statements are equivalent:

(1) $S$ is regular.

(2) $B=(B \circ S \circ B]$ for every bi-hyperideal $B$ of $S$.

(3) $B(a)=(B(a) \circ S \circ B(a)]$ for every $a \in S$.

\subsection{Basic concepts of soft sets}

In what follows, we take $E=S$ as the set of parameters, which is an ordered semihypergroup, unless otherwise specified.

From now on, $U$ is an initial universe set, $E$ is a set of parameters, $P(U)$ is the power set of $U$ and $A, B, C, \cdots \subseteq E$.

Definition 2.7. [24] A soft set $f_{A}$ over $U$ is defined as $f_{A}: E \longrightarrow P(U)$ such that $f_{A}(x)=\emptyset$ if $x \notin A$. Hence, $f_{A}$ is also called an approximation function.

A soft set $f_{A}$ over $U$ can be represented by the set of ordered pairs

$$
f_{A}=\left\{\left(x, f_{A}(x)\right) \mid x \in E, f_{A}(x) \in P(U)\right\} .
$$

It is clear from Definition 2.7, that a soft set is a parameterized family of subsets of $U$. Note that the set of all soft sets over $U$ will be denoted by $S(U)$.

Definition 2.8. [24] (i) Let $f_{A}, f_{B} \in S(U)$. Then $f_{A}$ is called a soft subset of $f_{B}$, denoted by $f_{A} \underset{\widetilde{\simeq}}{\widetilde{\subseteq}} f_{B}$ if $f_{A}(x) \subseteq f_{B}(x)$ for all $x \in E$. Two soft sets $f_{A}$ and $f_{B}$ are said to be equal soft sets if $f_{A} \widetilde{\subseteq} f_{B}$ and $f_{B} \widetilde{\subseteq} f_{A}$ and is denoted by $f_{A} \cong f_{B}$.

(ii) Let $f_{A}, f_{B} \in S(U)$. Then the soft union of $f_{A}$ and $f_{B}$, denoted by $f_{A} \widetilde{\cup} f_{B}=f_{A \cup B}$, is defined by $\left(f_{A} \widetilde{\cup} f_{B}\right)(x)=f_{A}(x) \cup f_{B}(x)$ for all $x \in E$. 
(iii) Let $f_{A}, f_{B} \in S(U)$. Then the soft intersection of $f_{A}$ and $f_{B}$, denoted by $f_{A} \widetilde{\cap} f_{B}=f_{A \cap B}$, is defined by $\left(f_{A} \widetilde{\cap} f_{B}\right)(x)=f_{A}(x) \cap f_{B}(x)$ for all $x \in E$.

(iv) $A \subseteq B$, if for any $x \in A$, we have, $f_{A}(x) \subseteq f_{B}(x)$. For $x \in S$, we define $A_{x}=\{(y, z) \in$ $S \times S \mid x \leq y \circ z\}$.

Definition 2.9. [17] Let $f_{A}$ and $g_{B}$ be two soft sets of an ordered semihypergroup $S$ over $U$. Then, the uni-soft product, denoted by $f_{A} \widetilde{*} g_{B}$, for all $x \in S$ is defined by

$$
f_{A} \widetilde{*} g_{B}: S \longrightarrow P(U), x \longmapsto\left(f_{A} \widetilde{*} g_{B}\right)(x)= \begin{cases}\bigcap_{(y, z) \in A_{x}}\left\{f_{A}(y) \cup g_{B}(z)\right\}, & \text { if } A_{x} \neq \emptyset, \\ U, & \text { if } A_{x}=\emptyset,\end{cases}
$$

Definition 2.10. 17] Let $A \subseteq S$. Then the soft characteristic function $\mathcal{S}_{A}: S \longrightarrow P(U)$ is defined by

$$
\mathcal{S}_{A}(x):=\left\{\begin{array}{l}
U \text { if } x \in A \\
\emptyset \text { if } x \notin A
\end{array}\right.
$$

The soft set $\left(U_{S}, S\right)$, where $U_{S}(x)=U$ for all $x \in S$, is called the identity soft set over $U$. For the characteristic soft set $\mathcal{S}_{A}$ over $U$, the soft set $\mathcal{S}_{A}^{c}$ over $U$ given as follows:

$$
\mathcal{S}_{A}^{c}(x):=\left\{\begin{array}{c}
\emptyset \text { if } x \in A \\
U \text { if } x \notin A .
\end{array}\right.
$$

For an ordered semihypergroup $S$, the soft set " $\mathcal{S}_{S}^{c}$ " of $S$ over $U$ is defined as follows:

$$
\mathcal{S}_{S}^{c}: S \longrightarrow P(U), x \longmapsto \mathcal{S}_{S}^{c}(x)=\emptyset \text { for all } x \in S .
$$

$\mathcal{S}_{S}^{c}$ is called an empty soft set of $S$ over $U$.

Definition 2.11. 17] Let $f_{A}$ be a soft set of an ordered semihypergroup $S$ over $U$ a subset $\delta$ such that $\delta \in P(U)$. The $\delta$-exclusive set of $f_{A}$ is denoted by $e_{A}\left(f_{A}, \delta\right)$ and defined to be the set

$$
e_{A}\left(f_{A}, \delta\right)=\left\{x \in S \mid f_{A}(x) \subseteq \delta\right\}
$$

Definition 2.12. [17] A soft set $f_{A}$ of an ordered semihypergroup $S$ over $U$ is called a uni-soft semihypergroup of $S$ over $U$ if for any $x, y \in S, \bigcup_{\alpha \in x \circ y} f_{A}(\alpha) \subseteq f_{A}(x) \cup f_{A}(y)$.

Definition 2.13. [17] Let $f_{A}$ be a soft set of an ordered semihypergroup $S$ over $U$. Then $f_{A}$ is called a uni-soft left (right) hyperideal of $S$ over $U$ if for all $x, y \in S$, it satisfies the following conditions:

$$
\bigcup_{\alpha \in x \circ y} f_{A}(\alpha) \subseteq f_{A}(y)\left(\bigcup_{\alpha \in x \circ y} f_{A}(\alpha) \subseteq f_{A}(x)\right) \text {. }
$$

(2) If $x \leq y$, then $f_{A}(x) \subseteq f_{A}(y)$.

A soft set $f_{A}$ of $S$ over $U$ is called a uni-soft hyperideal of $S$ over $U$ (or uni-soft two sided hyperideal) if it is both a uni-soft left hyperideal of $S$ over $U$ and a uni-soft right hyperideal of $S$ over $U$.

Definition 2.14. A uni-soft hyperideal $f_{A}$ of an ordered semihypergroup $S$ over $U$ is called idempotent if, $f_{A} \widetilde{*} f_{A}=f_{A}$. 


\section{Uni-soft bi-hyperideals}

Definition 3.1. A uni-soft semihypergroup $f_{A}$ of an ordered semihypergroup $S$ over $U$ is called a uni-soft bi-hyperideal of $S$ over $U$ if for all $x, y, z \in S$ it satisfies the following conditions:

$$
\bigcup_{\alpha \in x \circ y \circ z} f_{A}(\alpha) \subseteq f_{A}(x) \cup f_{A}(z)
$$

(2) If $x \leq y$, then $f_{A}(x) \subseteq f_{A}(y)$.

Example 3.2. Let $(S, \circ, \leq)$ be an ordered semihypergroup where the hyperoperation and order relation are defined:

\begin{tabular}{l|l|l|l|l|l|l}
$\circ$ & $a$ & $b$ & $c$ & $d$ & $e$ & $f$ \\
\hline$a$ & $\{a\}$ & $\{a\}$ & $\{a\}$ & $\{a\}$ & $\{a\}$ & $\{a\}$ \\
\hline$b$ & $\{a\}$ & $\{b\}$ & $\{b\}$ & $\{d\}$ & $\{b\}$ & $\{b\}$ \\
\hline$c$ & $\{a\}$ & $\{b\}$ & $\{c\}$ & $\{d\}$ & $\{e, f\}$ & $\{e, f\}$ \\
\hline$d$ & $\{a\}$ & $\{a\}$ & $\{d\}$ & $\{d\}$ & $\{d\}$ & $\{d\}$ \\
\hline$e$ & $\{a\}$ & $\{b\}$ & $\{c\}$ & $\{d\}$ & $\{e, f\}$ & $\{e, f\}$ \\
\hline$f$ & $\{a\}$ & $\{b\}$ & $\{c\}$ & $\{d\}$ & $\{e, f\}$ & $\{f\}$ \\
$\leq:=\{(a, a),(b, b),(c, c),(d, d),(e, e),(f, f),(f, e)\}$.
\end{tabular}

Suppose $U=\{p, q, r\}$ and $A=\{b, c, e, f\}$. Let us define $f_{A}(a)=\emptyset, f_{A}(b)=\{p\}, f_{A}(c)=\{p, q\}$, $f_{A}(d)=\emptyset, f_{A}(e)=\{p, q, r\}$ and $f_{A}(f)=\{p, q, r\}$. Then $f_{A}$ is a uni-soft bi-hyperideal of $S$ over $U$.

Proposition 3.3. [17] Let $S$ be an ordered semihypergroup such that $\mathcal{S}_{\mathcal{A}}^{c}$ and $\mathcal{S}_{\mathcal{B}}^{c}$ be soft sets of $S$ over $U$ where $A$ and $B$ are nonempty subsets of $S$. Then the following properties hold:

(1) $\mathcal{S}_{\mathcal{A}}^{c} \widetilde{\cup} \mathcal{S}_{B}^{c}=\mathcal{S}_{A \cup B}^{c}$

(2) $\mathcal{S}_{\mathcal{A}}^{c} \widetilde{*} \mathcal{S}_{\mathcal{B}}^{c}=\mathcal{S}_{(A \circ B]}^{c}$.

Proposition 3.4. [17] An ordered semihypergroup $S$ is intra-regular if and only if for every uni-soft right hyperideal $f_{A}$ and every uni-soft left hyperideal $g_{B}$ of $S$ over $U$, we have

$$
f_{A} \widetilde{\cup} g_{B} \widetilde{\supseteq} g_{B} \widetilde{*} f_{A} .
$$

Proposition 3.5. [17] Let $S$ be an ordered semihypergroup such that $f_{A}$ and $g_{B}$ be soft sets of $S$ over $U$. If $S$ is regular and $f_{A}$ is a uni-soft right hyperideal of $S$ over $U, g_{B}$ is a uni-soft left hyperideal of $S$ over $U$ then

$$
f_{A} \widetilde{\cup} g_{B} \widetilde{\supseteq} f_{A} \widetilde{*} g_{B}
$$

Equivalently, $f_{A} \widetilde{\cup} g_{B}=f_{A} \widetilde{*} g_{B}$.

Lemma 3.6. Let $S$ be an ordered semihypergroup, $f_{A}$ and $g_{B}$ be two soft sets of $S$ over $U$. Then

$$
\left(f_{A} \widetilde{*} g_{B}\right) \widetilde{\supseteq} f_{A} \widetilde{*} \mathcal{S}_{\mathcal{S}}^{c} \quad\left(\operatorname{resp} ., \quad\left(f_{A} \widetilde{*} g_{B}\right) \supseteq \widetilde{\mathcal{S}_{\mathcal{S}}} \widetilde{*}_{B}\right) .
$$

Proof. Let $a \in S$. If $A_{a}=\emptyset$, then $\left(f_{A} \widetilde{*} g_{B}\right)(a)=U \supseteq\left(f_{A} \widetilde{*} \mathcal{S}_{\mathcal{S}}^{c}\right)(a)$. Let $A_{a} \neq \emptyset$. Then

$$
\left(f_{A} \widetilde{*} g_{B}\right)(a)=\bigcap_{(y, z) \in A_{a}}\left\{f_{A}(y) \cup g_{B}(z)\right\}
$$


As $g_{B}(z) \supseteq \mathcal{S}_{\mathcal{S}}^{c}(z)$ for all $z \in S$. Thus

$$
\left(f_{A} \widetilde{*} g_{B}\right)(a)=\bigcap_{(y, z) \in A_{a}}\left\{f_{A}(y) \cup g_{B}(z)\right\} \supseteq \bigcap_{(y, z) \in A_{a}}\left\{f_{A}(y) \cup \mathcal{S}_{\mathcal{S}}^{c}(z)\right\}=\left(f_{A} \widetilde{*} \mathcal{S}_{\mathcal{S}}^{c}\right)(a) .
$$

Theorem 3.7. Let $(S, \circ, \leq)$ be an ordered semihypergroup and $B$ be a nonempty subset of $S$. Then $B$ is bi-hyperideal of $S$ if and only if the soft set $\mathcal{S}_{\mathcal{B}}^{c}$ is a uni-soft bi-hyperideal of $S$ over $U$.

Proof. Suppose that $B$ is a bi-hyperideal of $S$. Let $x, y \in S$ such that $x \leq y$. If $y \notin B$, then $\mathcal{S}_{\mathcal{B}}^{c}(y)=$ $U$ and so $\mathcal{S}_{\mathcal{B}}^{c}(x) \subseteq U=\mathcal{S}_{\mathcal{B}}^{c}(y)$. If $y \in B$, then $\mathcal{S}_{\mathcal{B}}^{c}(y)=\emptyset$. Since $x \leq y$ and $B$ is a bi-hyperideal of $S$, we have $x \in B$ and thus $\mathcal{S}_{\mathcal{B}}^{c}(x)=\emptyset=\mathcal{S}_{\mathcal{B}}^{c}(y)$. Therefore, $B$ is a subsemihypergroup of $S$. Let $x, y \in S$. Then $\bigcup_{\alpha \in x \circ y} \mathcal{S}_{\mathcal{B}}^{c}(\alpha) \subseteq \mathcal{S}_{\mathcal{B}}^{c}(x) \cup \mathcal{S}_{\mathcal{B}}^{c}(y)$ for every $\alpha \in x \circ y$. Indeed: If $x \circ y \nsubseteq B$, then there exists $\alpha \in x \circ y$ such that $\alpha \notin B, \mathcal{S}_{\mathcal{B}}^{c}(\alpha)=U$, so we have $\bigcup_{\alpha \in x \circ y} \mathcal{S}_{\mathcal{B}}^{c}(\alpha)=U$. Besides that $x \circ y \nsubseteq B$ implies that $x \notin B$ or $y \notin B$. Then $\mathcal{S}_{\mathcal{B}}^{c}(x)=U$ or $\mathcal{S}_{\mathcal{B}}^{c}(y)=U$ and so $\bigcup_{\alpha \in x \circ y} \mathcal{S}_{\mathcal{B}}^{c}(\alpha)=\mathcal{S}_{\mathcal{B}}^{c}(x) \cup \mathcal{S}_{\mathcal{B}}^{c}(y)$. Let $x \circ y \subseteq B$. Then $\mathcal{S}_{\mathcal{B}}^{c}(\alpha)=\emptyset$ for any $\alpha \in x \circ y$. It implies that $\bigcup_{\alpha \in x \circ y} \mathcal{S}_{\mathcal{B}}^{c}(\alpha)=\emptyset$. Since $\mathcal{S}_{\mathcal{B}}^{c}(x) \supseteq \emptyset$ for any $x \in B$, we have $\bigcup_{\alpha \in x \circ y} \mathcal{S}_{\mathcal{B}}^{c}(\alpha) \subseteq \mathcal{S}_{\mathcal{B}}^{c}(x) \cup \mathcal{S}_{\mathcal{B}}^{c}(y)$. Therefore, $\mathcal{S}_{\mathcal{B}}^{c}$ is a uni-soft semihypergroup of $S$ over $U$. Let $x, y$ and $z$ be any elements of $S$. If $x, z \in B$, then $\mathcal{S}_{\mathcal{B}}^{c}(x)=\mathcal{S}_{\mathcal{B}}^{c}(z)=\emptyset$ and since for every $\alpha \in x \circ y \circ z \subseteq B \circ S \circ B \subseteq B$, we have $\mathcal{S}_{\mathcal{B}}^{c}(\alpha)=\emptyset=\left\{\mathcal{S}_{\mathcal{B}}^{c}(x) \cup \mathcal{S}_{\mathcal{B}}^{c}(z)\right\}$. Thus $\bigcup_{\alpha \in x \circ y \circ z} \mathcal{S}_{\mathcal{B}}^{c}(\alpha)=\emptyset=\left\{\mathcal{S}_{\mathcal{B}}^{c}(x) \cup \mathcal{S}_{\mathcal{B}}^{c}(z)\right\}$. If $x \notin B$ or $z \notin B$, then $\mathcal{S}_{\mathcal{B}}^{c}(x)=U$ or $\mathcal{S}_{\mathcal{B}}^{c}(z)=U$, and so we have $\mathcal{S}_{\mathcal{B}}^{c}(\alpha) \subseteq U=\left\{\mathcal{S}_{\mathcal{B}}^{c}(x) \cup \mathcal{S}_{\mathcal{B}}^{c}(z)\right\}$. Thus $\bigcup_{\alpha \in x \circ y \circ z} \mathcal{S}_{\mathcal{B}}^{c}(\alpha) \subseteq\left\{\mathcal{S}_{\mathcal{B}}^{c}(x) \cup \mathcal{S}_{\mathcal{B}}^{c}(z)\right\}$. Therefore, $\mathcal{S}_{\mathcal{B}}^{c}$ is a uni-soft bi-hyperideal of $S$ over $U$.

Conversely, let $\emptyset \neq B \subseteq S$ such that $\mathcal{S}_{\mathcal{B}}^{c}$ is a uni-soft bi-hyperideal of $S$ over $U$. We claim that $B \circ B \subseteq B$. To prove the claim, let $x, y \in B$. By hypothesis, $\bigcup_{\alpha \in x \circ y} \mathcal{S}_{\mathcal{B}}^{c}(\alpha) \subseteq \mathcal{S}_{\mathcal{B}}^{c}(x) \cup \mathcal{S}_{\mathcal{B}}^{c}(y)=\emptyset$ which implies that $\mathcal{S}_{\mathcal{B}}^{c}(\alpha) \subseteq \emptyset$ for any $\alpha \in x \circ y$. On the other hand $\mathcal{S}_{\mathcal{B}}^{c}(x) \supseteq \emptyset$ for all $x \in S$. Thus for any $\alpha \in x \circ y, \mathcal{S}_{\mathcal{B}}^{c}(\alpha)=\emptyset$ implies that $\alpha \in B$. It follows that $B \circ B \subseteq B$. Hence, $B$ is a subsemihypergroup of $S$. Let $\alpha \in B \circ S \circ B$, then there exist $x, z \in B$ and $y \in S$ such that $\alpha \in x \circ y \circ z$. Since

$$
\bigcup_{\alpha \in x \circ y \circ z} \mathcal{S}_{\mathcal{B}}^{c}(\alpha) \subseteq \mathcal{S}_{\mathcal{B}}^{c}(x) \cup \mathcal{S}_{\mathcal{B}}^{c}(z)=\emptyset \cup \emptyset=\emptyset
$$

Hence, for each $\alpha \in x \circ y \circ z$, we have $\mathcal{S}_{\mathcal{B}}^{c}(\alpha)=\emptyset$, and so $\alpha \in B$. Thus $B \circ S \circ B \subseteq B$. Let $x \in S$ and $y \in B$ such that $x \leq y$. Then $\mathcal{S}_{\mathcal{B}}^{c}(x) \subseteq \mathcal{S}_{\mathcal{B}}^{c}(y)=\emptyset$, and so $x \in B$. Thus $B$ is a bi-hyperideal of $S$.

Theorem 3.8. Let $f_{A}$ be a soft set of an ordered semihypergroup $S$ over $U$ and $\delta \in P(U)$. Then $f_{A}$ is a uni-soft bi-hyperideal of $S$ over $U$ if and only if nonempty $\delta$-exclusive set $e_{A}\left(f_{A}, \delta\right)$ is a bi-hyperideal of $S$. 
Proof. Assume that $f_{A}$ is a uni-soft bi-hyperideal of $S$ over $U$. Suppose $\delta \in P(U)$ with $e_{A}\left(f_{A}, \delta\right) \neq$ $\emptyset$. Let $a \in e_{A}\left(f_{A}, \delta\right) \circ S \circ e_{A}\left(f_{A}, \delta\right)$, for any $a \in b \circ s \circ c$ for some $b, c \in e_{A}\left(f_{A}, \delta\right)$ and $s \in S$. Since $\delta \supseteq f_{A}(b) \cup f_{A}(c) \supseteq \bigcup_{a \in \text { bosoc }} f_{A}(a)$, we have $f_{A}(a) \subseteq \delta$. We get $a \in e_{A}\left(f_{A}, \delta\right)$. This implies that $e_{A}\left(f_{A}, \delta\right) \circ S \circ e_{A}\left(f_{A}, \delta\right) \subseteq e_{A}\left(f_{A}, \delta\right)$. Let $x \in e_{A}\left(f_{A}, \delta\right)$ and $y \in S$ with $y \leq x$. Since $\delta \supseteq f_{A}(x) \supseteq$ $f_{A}(y)$, we get $y \in e_{A}\left(f_{A}, \delta\right)$. Therefore, $e_{A}\left(f_{A}, \delta\right)$ is a bi-hyperideal of $S$.

Conversely, we assume that for every $\delta \in P(U), e_{A}\left(f_{A}, \delta\right)$ is a bi-hyperideal of $S$. We show that $\bigcup_{a \in b \circ s \circ c} f_{S}(a) \subseteq f_{A}(b) \cup f_{A}(c)$ for all $b, s, c \in S$. We choose $\delta=f_{A}(b) \cup f_{A}(c)$. By assumption $e_{A}\left(f_{A}, \delta\right)$ is a bi-hyperideal of $S$. Since $b, c \in e_{A}\left(f_{A}, \delta\right), b \circ s \circ c \subseteq e_{A}\left(f_{A}, \delta\right)$. Then for every $a \in b \circ s \circ c$, we have $f_{A}(a) \subseteq \delta$ and so $\bigcup f_{A}(a) \subseteq \delta=f_{A}(b) \cup f_{A}(c)$. Let $a, b \in S$ such that $a \leq b$. Since $a \leq b, b \in e_{A}\left(f_{A}, \delta\right)$ let $f_{A}(b)=\delta$ and $e_{A}\left(f_{A}, \delta\right)$ is a bi-hyperideal of $S$, we get $a \in e_{A}\left(f_{A}, \delta\right)$. So $f_{A}(a) \subseteq \delta=f_{A}(b)$. Hence, $f_{A}(a) \subseteq f_{A}(b)$. Therefore, $f_{A}$ is a uni-soft bi-hyperideal of $S$ over $U$.

Example 3.9. Let $(S, \circ, \leq)$ be an ordered semihypergroup where the hyperoperation and the order relation are defined by:

\begin{tabular}{r|l|l|l}
$\circ$ & $a$ & $b$ & $c$ \\
\hline$a$ & $\{a\}$ & $\{a, b\}$ & $\{a, c\}$ \\
\hline$b$ & $\{a\}$ & $\{a, b\}$ & $\{a, c\}$ \\
\hline$c$ & $\{a\}$ & $\{a, b\}$ & $\{c\}$ \\
$\leq:=$ & $\{(a, a),(b, b),(c, c),(a, b)\}$.
\end{tabular}

Suppose $U=\{1,2,3\}$ and $A=\{b, c\}$. Here $\{a\},\{a, b\},\{a, c\}$, and $S$ are bi-hyperideals of $S$. Let us define $f_{A}(a)=\emptyset, f_{A}(b)=\{1,2\}$, and $f_{A}(c)=\{1,3\}$. Then

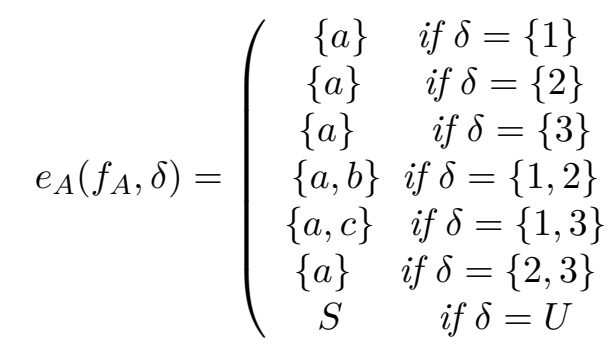

So by Theorem 3.8, $f_{A}$ is a uni-soft bi-hyperideal of $S$ over $U$.

Proposition 3.10. Let $f_{B}$ be a uni-soft bi-hyperideal of an ordered semihypergroup $S$ over $U$. Then:

(1) $f_{B} \widetilde{*} f_{B} \supseteq f_{B}$;

(2) $f_{B} \widetilde{*} \mathcal{S}_{\mathcal{S}}^{c} \widetilde{\sim} f_{B} \supseteq f_{B}$.

Proof. (1) Let $f_{B}$ be a uni-soft bi-hyperideal of an ordered semihypergroup $S$ over $U$. Then for each $x, y, z \in S$, if $A_{x}=\emptyset$, then $\left(f_{B} \widetilde{*} f_{B}\right)(x)=U \supseteq f_{B}(x)$. If $A_{x} \neq \emptyset$, then

$$
\left(f_{B} \widetilde{*} f_{B}\right)(x)=\bigcap_{(y, z) \in A_{x}}\left\{f_{B}(y) \cup f_{B}(z)\right\} .
$$

As $f_{B}$ is a uni-soft semihypergroup of $S$ over $U$, we have $\bigcup_{x \in y \circ z} f_{B}(x) \subseteq f_{B}(y) \cup f_{B}(z)$ for each $y, z \in S, x \in y \circ z$. Thus $f_{B}(x) \subseteq f_{B}(y) \cup f_{B}(z)$. Hence,

$$
\left(f_{B} \widetilde{*} f_{B}\right)(x)=\bigcap_{(y, z) \in A_{x}}\left\{f_{B}(y) \cup f_{B}(z)\right\} \supseteq f_{B}(x) .
$$


Thus, $f_{B} \widetilde{*} f_{B} \widetilde{\supseteq} f_{B}$.

(2) Let $a \in S$. If $A_{a}=\emptyset$, then $\left(f_{B} \widetilde{*} \mathcal{S}_{\mathcal{S}}^{c} \widetilde{*} f_{B}\right)(a)=U \supseteq f_{B}(a)$. Let $A_{a} \neq \emptyset$. Then

$$
\begin{aligned}
\left(f_{B} \widetilde{*} \mathcal{S}_{\mathcal{S}}^{c} \widetilde{*} f_{B}\right)(a) & =\bigcap_{(y, z) \in A_{a}}\left\{f_{B}(y) \cup\left(\mathcal{S}_{\mathcal{S}}^{c} \widetilde{*} f_{B}\right)(z)\right\} \\
& =\bigcap_{(y, z) \in A_{a}}\left\{f_{B}(y) \cup\left\{\bigcap_{(p, q) \in A_{z}}\left(\mathcal{S}_{\mathcal{S}}^{c}(p) \cup f_{B}(q)\right)\right\}\right\} \\
& =\bigcap_{(y, z) \in A_{a}(p, q) \in A_{z}}\left\{f_{B}(y) \cup\left(\mathcal{S}_{\mathcal{S}}^{c}(p) \cup f_{B}(q)\right)\right\} \\
& =\bigcap_{(y, z) \in A_{a}(p, q) \in A_{z}}\left\{f_{B}(y) \cup\left(\emptyset \cup f_{B}(q)\right)\right\} \\
& =\bigcap_{(y, z) \in A_{a}(p, q) \in A_{z}}\left\{f_{B}(y) \cup f_{B}(q)\right\} .
\end{aligned}
$$

Since $(y, z) \in A_{a}$ and $(p, q) \in A_{z}$ we have $a \leq y \circ z$ and $z \leq p \circ q$, respectively. Thus $a \leq y \circ z \leq y \circ(p \circ q)$ implies that $a \leq y \circ p \circ q$. So there exists $\beta \in y \circ p \circ q$ such that $a \leq \beta$. Since $f_{B}$ is a uni-soft bi-hyperideal of $S$ over $U$, we get

$$
f_{B}(a) \subseteq f_{B}(\beta) \subseteq \bigcup_{\beta \in y \circ p \circ q} f_{B}(\beta) \subseteq f_{B}(y) \cup f_{B}(q) \text { for all } y, p, q \in S
$$

Thus

$$
\bigcap_{(y, z) \in A_{a}} \bigcap_{(p, q) \in A_{z}}\left\{f_{B}(y) \cup f_{B}(q)\right\} \supseteq \bigcap_{(y, z) \in A_{a}(p, q) \in A_{z}} \bigcap_{B}(a)=f_{B}(a) .
$$

Therefore, $f_{B} \widetilde{*} \mathcal{S}_{\mathcal{S}}^{c} \widetilde{*} f_{B} \supseteq f_{B}$.

Theorem 3.11. An ordered semihypergroup $S$ is regular if and only if $f_{B} \widetilde{*} \mathcal{S}_{\mathcal{S}}^{c} \widetilde{*} f_{B}=f_{B}$, for every uni-soft bi-hyperideal $f_{B}$ of $S$ over $U$.

Proof. Let $S$ be an ordered semihypergroup, $f_{B}$ is a uni-soft bi-hyperideal of $S$ over $U$ and $a \in S$. Since $S$ is regular, there exists $x \in S$ such that $a \leq a \circ x \circ a \leq a \circ x \circ(a \circ x \circ a)=a \circ(x \circ a \circ x \circ a)$ such that $a \leq a \circ z$ for some $z \in x \circ a \circ x \circ a$. Since $A_{a} \neq \emptyset$, we get

$$
\left(f_{B} \widetilde{*} \mathcal{S}_{\mathcal{S}}^{c} \widetilde{*} f_{B}\right)(a)=\bigcap_{(y, z) \in A_{a}}\left\{f_{B}(y) \cup\left(\mathcal{S}_{\mathcal{S}}^{c} \widetilde{*} f_{B}\right)(z)\right\} \subseteq\left\{f_{B}(a) \cup\left(\mathcal{S}_{\mathcal{S}}^{c} \widetilde{*} f_{B}\right)(z)\right\} .
$$

Now, since $z \in x \circ a \circ x \circ a$, there exists $\alpha \in x \circ a \circ x$ such that $z \leq \alpha \circ a$ and $A_{z} \neq \emptyset$. Then

$$
\left(\mathcal{S}_{\mathcal{S}}^{c} \widetilde{*} f_{B}\right)(z)=\bigcap_{(p, q) \in A_{z}}\left\{\mathcal{S}_{\mathcal{S}}^{c}(p) \cup f_{B}(q)\right\} \subseteq\left\{\mathcal{S}_{\mathcal{S}}^{c}(\alpha) \cup f_{B}(a)\right\}=\emptyset \cup f_{B}(a)=f_{B}(a)
$$

Thus,

$$
f_{B} \widetilde{*} \mathcal{S}_{\mathcal{S}}^{c} \widetilde{*} f_{B} \widetilde{\subseteq} f_{B}(a) \cup f_{B}(a)=f_{B}(a) .
$$

On the other hand by Proposition 3.10, for every uni-soft bi-hyperideal $f_{B}$ of $S$ over $U$, we have $f_{B} \widetilde{*} \mathcal{S}_{\mathcal{S}}^{c} \widetilde{*} f_{B} \widetilde{\supseteq} f_{B}$. Hence, $f_{B} \widetilde{*} \mathcal{S}_{\mathcal{S}}^{c} \widetilde{*} f_{B}=f_{B}$. 
Conversely, assume that $f_{B} \widetilde{*} \mathcal{S}_{\mathcal{S}}^{c} \widetilde{*} f_{B}=f_{B}$ for every uni-soft bi-hyperideal $f_{B}$ of $S$ over $U$. Then $S$ is regular. In fact, by Lemma 2.6, it is enough to prove that

$$
B(a)=(B(a) \circ S \circ B(a)], \text { for all } a \in S .
$$

Let $y \in B(a)$. Then $y \in(B(a) \circ S \circ B(a)]$. Indeed: Since $B(a)$ is the bi-hyperideal of $S$ generated $a$, by Theorem 3.7, $\mathcal{S}_{B(a)}^{c}$ is a uni-soft bi-hyperideal of $S$ over $U$. By hypothesis,

$$
\left(\mathcal{S}_{B(a)}^{c} \widetilde{*} \mathcal{S}_{\mathcal{S}}^{c} \widetilde{S_{B(a)}^{c}}\right)(y)=\mathcal{S}_{B(a)}^{c}(y) .
$$

As $y \in B(a)$, we have $\mathcal{S}_{B(a)}^{c}(y)=\emptyset$. Hence, $\left(\mathcal{S}_{B(a)}^{c} \widetilde{*} \mathcal{S}_{\mathcal{S}}^{c} \widetilde{*} \mathcal{S}_{B(a)}^{c}\right)(y)=\emptyset$. By Proposition 3.3 , $\mathcal{S}_{B(a)}^{c} \widetilde{*} \mathcal{S}_{\mathcal{S}}^{c} \widetilde{*} \mathcal{S}_{B(a)}^{c}=\mathcal{S}_{(B(a) \circ S \circ B(a)]}^{c}$. Thus $\mathcal{S}_{(B(a) \circ S \circ B(a)]}^{c}(y)=\emptyset$ implies $y \in(B(a) \circ S \circ B(a)]$. On the other hand $(B(a) \circ S \circ B(a)] \subseteq(B(a)]=B(a)$. Therefore, $(B(a) \circ S \circ B(a)]=B(a)$.

Proposition 3.12. A soft set $f_{A}$ is a uni-soft semihypergroup of an ordered semihypergroup $S$ over $U$ if and only if $f_{A} \widetilde{*} f_{A} \supseteq f_{A}$.

Proof. Straightforward.

Lemma 3.13. Let $S$ be an ordered semihypergroup, $f_{A}, g_{B}$ be two uni-soft bi-hyperideals of $S$ over $U$. Then $f_{A} \widetilde{*} g_{B}$ is a uni-soft bi-hyperideal of $S$ over $U$.

Proof. Straightforward.

\section{Prime and semiprime uni-soft bi-hyperideals of ordered semi- hypergroups.}

In this section, we define prime, strongly prime, semiprime, irreducible and strongly irreducible uni-soft bi-hyperideals of ordered semihypergroups. We characterize ordered semihypergroups by the properties of these notions.

Definition 4.1. Let $(S, \circ, \leq)$ be an ordered semihypergroup and $f_{A}$ be a uni-soft bi-hyperideal of $S$ over $U . f_{A}$ is called prime (resp., strongly prime, semiprime) if $h_{D} \widetilde{\supseteq} f_{A}\left(\right.$ resp., $g_{C} \cong f_{A}$ or $\left.h_{D} \cong f_{A}, g_{C} \cong f_{A}\right)$ or $g_{C} \widetilde{*} h_{D} \supseteq f_{A}\left(\right.$ resp., $\left.\left(g_{C} \widetilde{*} h_{D}\right) \widetilde{\cup}\left(h_{D} \widetilde{*} g_{C}\right) \supseteq f_{A}, g_{C} \widetilde{*} g_{C} \supseteq f_{A}\right)$, then $g_{C} \supseteq f_{A}$ for all uni-soft bi-hyperideals $g_{C}$ and $h_{D}$ of $S$ over $U$.

Example 4.2. Let $U=\left\{h_{1}, h_{2}, h_{3}\right\}$ be a set of houses under consideration where $E:=\left\{e_{1}, e_{2}, e_{3}\right\}$ be the set of parameters for selection of the house. Let

$e_{1}$ stands for "expensive houses",

$e_{2}$ stands for "wooden houses",

$e_{3}$ stands for "houses located in the urban area",

with the following binary operation o given in the Cayley table:

$$
\begin{array}{r|l|l|l}
\circ & e_{1} & e_{2} & e_{3} \\
\hline e_{1} & \left\{e_{1}\right\} & \left\{e_{1}\right\} & \left\{e_{1}\right\} \\
\hline e_{2} & \left\{e_{1}\right\} & \left\{e_{1}, e_{2}\right\} & \left\{e_{1}, e_{2}\right\} \\
\hline e_{3} & \left\{e_{1}\right\} & \left\{e_{1}, e_{3}\right\} & \left\{e_{1}, e_{3}\right\} \\
\leq:=\left\{\left(e_{1}, e_{1}\right),\left(e_{2}, e_{2}\right),\left(e_{3}, e_{3}\right),\left(e_{1}, e_{2}\right),\left(e_{1}, e_{3}\right)\right\} .
\end{array}
$$


The covering relation is given as follows, $\prec:=\left\{\left(e_{1}, e_{2}\right),\left(e_{1}, e_{3}\right)\right\}$. Then $(E, \circ, \leq)$ is an ordered semihypergroup.

Suppose $A=\left\{e_{2}, e_{3}\right\}$. Let us define $f_{A}\left(e_{1}\right)=\emptyset, f_{A}\left(e_{2}\right)=\left\{h_{1}, h_{2}\right\}$ and $f_{A}\left(e_{3}\right)=\left\{h_{1}, h_{2}, h_{3}\right\}$. Then $f_{A}$ is a uni-soft bi-hyperideal of $S$ over $U$. Now, take $B=\left\{e_{2}\right\}$. Let us define $g_{B}\left(e_{1}\right)=\emptyset$, $g_{B}\left(e_{2}\right)=\left\{h_{1}, h_{3}\right\}$ and $g_{B}\left(e_{3}\right)=\emptyset$. Then $g_{B}$ is also a uni-soft bi-hyperideal of $S$ over $U$.

Also, $f_{A} \widetilde{*} g_{B} \supseteq g_{B}$ for every uni-soft bi-hyperideals $f_{A}$ and $g_{B}$ of $S$ over $U$. Thus every uni-soft bi-hyperideal is prime. Hence, every uni-soft bi-hyperideal is semiprime. Also, $\left(f_{A} \widetilde{*} g_{B}\right)\left(e_{2}\right)=$ $\left\{h_{1}, h_{2}, h_{3}\right\}$

Proposition 4.3. Let $\left\{f_{B_{i}}: i \in I\right\}$ be a family of prime uni-soft bi-hyperideals of an ordered semihypergroup $S$ over $U$. Then $\bigcup_{i \in I} f_{B_{i}}$ is a semiprime uni-soft bi-hyperideal of $S$ over $U$.

Proof. Since union of uni-soft bi-hyperideals is a uni-soft bi-hyperideal, we get $\bigcup_{i \in I} f_{B_{i}}$ is a uni-soft bi-hyperideal of $S$ over $U$. Let $g_{C}$ be any uni-soft bi-hyperideal of $S$ over $U$ such that $g_{C} \widetilde{*} g_{C} \widetilde{\supseteq} \bigcup_{i \in I} f_{B_{i}}$. Then $g_{C} \widetilde{*} g_{C} \supseteq f_{B_{i}}$ for all $i \in I$. Since each $f_{B_{i}}$ is prime uni-soft bi-hyperideal of $S$ over $U$. So $g_{C} \widetilde{\supseteq} f_{B_{i}}$ for all $i \in I$. Hence, $g_{C} \widetilde{\supseteq} \bigcup_{i \in I} f_{B_{i}}$. Thus $\bigcup_{i \in I} f_{B_{i}}$ is a semiprime uni-soft bi-hyperideal of $S$ over $U$.

Definition 4.4. Let $(S, \circ, \leq)$ be an ordered semihypergroup. A uni-soft bi-hyperideal $f_{B}$ of $S$ over $U$ is called irreducible (resp., strongly irreducible) uni-soft bi-hyperideal of $S$ over $U$ if for any unisoft bi-hyperideals $g_{C}$ and $h_{D}$ of $S$ over $U, g_{C} \widetilde{\cup} h_{D}=f_{B}\left(\right.$ resp., $\left.g_{C} \widetilde{\cup} h_{D} \widetilde{\supseteq} f_{B}\right)$ implies either $g_{C}=$ $f_{B}$ or $h_{D}=f_{B}\left(\right.$ resp., $g_{C} \supseteq f_{B}$ or $\left.h_{D} \cong f_{B}\right)$.

Theorem 4.5. Every strongly irreducible semiprime uni-soft bi-hyperideal of an ordered semihypergroup $S$ over $U$ is a strongly prime uni-soft bi-hyperideal of $S$ over $U$.

Proof. Let $f_{B}$ be a strongly irreducible semiprime uni-soft bi-hyperideal of $S$ over $U$. Let $g_{C}$ and $h_{D}$ be any two uni-soft bi-hyperideals of $S$ over $U$ such that

$$
\left(g_{C} \widetilde{*} h_{D}\right) \widetilde{\cup}\left(h_{D} \widetilde{*} g_{C}\right) \widetilde{\supseteq} f_{B} .
$$

As $g_{C} \widetilde{\cup} h_{D}$ is a uni-soft bi-hyperideal of $S$ over $U$, and $\left(g_{C} \widetilde{\cup} h_{D}\right) \widetilde{*}\left(h_{D} \widetilde{\cup} g_{C}\right) \widetilde{\supseteq} g_{C} \widetilde{*} h_{D}$ and

$$
\left(g_{C} \widetilde{\cup} h_{D}\right) \widetilde{*}\left(h_{D} \widetilde{\cup} g_{C}\right) \supseteq h_{D} \widetilde{*} g_{C} .
$$

Thus

$$
\left(g_{C} \widetilde{\cup} h_{D}\right) \widetilde{*}\left(h_{D} \widetilde{\cup} g_{C}\right) \simeq\left(g_{C} \widetilde{*} h_{D}\right) \widetilde{\cup}\left(h_{D} \widetilde{*} g_{C}\right) \widetilde{\supseteq} f_{B} .
$$

Since $f_{B}$ is a semiprime uni-soft bi-hyperideal of $S$ over $U$, we obtain $g_{C} \widetilde{\cup} h_{D} \simeq f_{B}$. As $f_{B}$ is strongly irreducible uni-soft bi-hyperideal of $S$ over $U$, so either $g_{C} \cong f_{B}$ or $h_{D} \supseteq f_{B}$. Thus $f_{B}$ is a strongly prime uni-soft bi-hyperideal of $S$ over $U$.

Proposition 4.6. Let $f_{B}$ be a uni-soft bi-hyperideal of an ordered semihypergroup $S$ over $U$ with $f_{B}(a)=t$ where $a \in S$ and $t \in P(U)$. Then there exists an irreducible uni-soft bi-hyperideal $g_{C}$ of $S$ over $U$ such that $f_{B} \widetilde{\supseteq} g_{C}$ and $g_{C}(a)=t$. 
Proof. Let $X=\left\{h_{D}: h_{D}\right.$ is a uni-soft bi-hyperideal of $S$ over $U$ with $h_{D}(a)=t$ and $\left.f_{B} \cong h_{D}\right\}$. Then $X \neq \emptyset$, since $f_{B} \in X$. The collection $X$ is partially ordered set under inclusion. Let $Y$ be any totally ordered subset of $X$, say $Y=\left\{h_{D_{i}}: i \in I\right\}$. Let $x, y, z \in S$ and for every $\alpha \in x \circ y$,

$$
\begin{aligned}
& \bigcup_{\alpha \in x \circ y}\left(\bigcap_{i \in I} h_{D_{i}}\right)(\alpha)=\bigcap_{i \in I}\left(\bigcup_{\alpha \in x \circ y} h_{D_{i}}(\alpha)\right) \subseteq \bigcap_{i \in I}\left(h_{D_{i}}(x) \cup h_{D_{i}}(y)\right) \\
& =\left\{\bigcap_{i \in I}\left(h_{D_{i}}(x)\right)\right\} \cup\left\{\bigcap_{i \in I}\left(h_{D_{i}}(y)\right)\right\}=\left\{\bigcap_{i \in I} h_{D_{i}}\right\}(x) \cup\left\{\bigcup_{i \in I} h_{D_{i}}\right\}(y) .
\end{aligned}
$$

Hence $\bigcap_{i \in I} h_{D_{i}}$ is a uni-soft semihypergroup of $S$ over $U$.

Also, for any $\gamma \in x \circ y \circ z$,

$$
\begin{aligned}
\bigcup_{\gamma \in x \circ y \circ z}\left(\bigcap_{i \in I} h_{D_{i}}\right)(\gamma) & =\bigcap_{i \in I}\left(\bigcup_{\gamma \in x \circ y \circ z} h_{D_{i}}(\gamma)\right) \subseteq \bigcap_{i \in I}\left(h_{D_{i}}(x) \cup h_{D_{i}}(z)\right) \\
& =\left\{\bigcap_{i \in I}\left(h_{D_{i}}(x)\right)\right\} \cup\left\{\bigcap_{i \in I}\left(h_{D_{i}}(z)\right)\right\}=\left\{\bigcap h_{D_{i}}\right\}(x) \cup\left\{\bigcap h_{i \in I}\right\}(z) .
\end{aligned}
$$

Let $x, y \in S$ such that $x \leq y$. Since $h_{D_{i}}$ are uni-soft bi-hyperideal of $S$ over $U$, we get

$$
\left(\bigcap_{i \in I} h_{D_{i}}\right)(x)=\bigcap_{i \in I}\left(h_{D_{i}}(x)\right) \subseteq \bigcap_{i \in I}\left(h_{D_{i}}(y)\right)=\left(\bigcap_{i \in I} h_{D_{i}}\right)(y) .
$$

Hence, $\bigcap_{i \in I} h_{D_{i}}$ is a uni-soft bi-hyperideal of $S$ over $U$. As $f_{B} \widetilde{\supseteq} h_{D_{i}}$ for each $i \in I$, so $f_{B} \widetilde{\supseteq} \bigcap_{i \in I} h_{D_{i}}$. Also, $\left(\bigcap_{i \in I} h_{D_{i}}\right)(a)=\bigcap_{i \in I}\left(h_{D_{i}}(a)\right)=t$. Thus $\bigcap_{i \in I} h_{D_{i}}$ is the greatest lower bound of $Y$. By Zorn's Lemma, there exists a uni-soft bi-hyperideal $g_{C}$ of $S$ over $U$, which is minimal with respect to the property that $f_{B} \widetilde{\supseteq} g_{C_{i}}$ and $g_{C}(a)=t$. Now, we show that $g_{C}$ is an irreducible uni-soft bi-hyperideal of $S$ over $U$. Suppose $g_{C}=g_{C_{1}} \widetilde{\cup} g_{C_{2}}$ where $g_{C_{1}}$ and $g_{C_{2}}$ are uni-soft bi-hyperideal of $S$ over $U$. Then $g_{C} \supseteq g_{C_{1}}$ and $g_{C} \widetilde{\supseteq} g_{C_{2}}$. We claim that $g_{C}=g_{C_{1}}$ or $g_{C}=g_{C_{2}}$. Suppose on contrary that $g_{C} \neq g_{C_{1}}$ and $g_{C} \neq g_{C_{2}}$. Since $g_{C}$ is minimal with respect to the property that $g_{C}(a)=t$ and since $g_{C} \neq g_{C_{1}}$ and $g_{C} \neq g_{C_{2}}$, it follows that $g_{C_{1}}(a) \neq t$ and $g_{C_{2}}(a) \neq t$. Thus $t=g_{C}(a)=\left(g_{C_{1}} \widetilde{\cup} g_{C_{2}}\right)(a) \neq t$, which is a contradiction. Hence, either $g_{C}=g_{C_{1}}$ or $g_{C}=g_{C_{2}}$. Thus $g_{C}$ is an irreducible uni-soft bi-hyperideal of $S$ over $U$.

Theorem 4.7. An ordered semihypergroup $S$ is both regular and intra-regular if and only if for every uni-soft right hyperideal $f_{A}$ of $S$ over $U$ and every uni-soft left hyperideal $g_{B}$ of $S$ over $U$, we have

$$
f_{A} \widetilde{\cup} g_{B} \widetilde{\supseteq}\left(g_{B} \widetilde{*} f_{A}\right) \widetilde{\cup}\left(f_{A} \widetilde{*} g\right) .
$$

Proof. Follows from Propositions 3.4 and 3.5 . 
Theorem 4.8. For an ordered semihypergroup $S$ the following statements are equivalent:

(1) $S$ is both regular and intra-regular.

(2) $f_{B}=f_{B} \widetilde{*} f_{B}$ for every uni-soft bi-hyperideal $f_{B}$ of $S$ over $U$.

(3) $g_{C} \widetilde{\cup} h_{D}=\left(g_{C} \widetilde{*} h_{D}\right) \widetilde{\cup}\left(h_{D} \widetilde{*} g_{C}\right)$ for every uni-soft bi-hyperideal $g_{C}$ and $h_{D}$ of $S$ over $U$.

(4) Each uni-soft bi-hyperideal of $S$ over $U$ is semiprime.

(5) Each proper uni-soft bi-hyperideal of $S$ over $U$ is the union of all irreducible semiprime uni-soft bi-hyperideals of $S$ over $U$ which contained in.

Proof. (1) $\Leftrightarrow(2)$. Suppose $S$ is both regular and intra-regular ordered semihypergroup and $f_{B}$ is a uni-soft bi-hyperideal of $S$ over $U$. Then for each $a \in S$, we have $\left(f_{B} \widetilde{*} f_{B}\right)(a) \widetilde{\subseteq} f_{B}(a)$. Indeed: Since $S$ is regular and intra-regular, we obtain there exist $x, y, z \in S$ such that $a \leq a \circ x \circ a$ and $a \leq y \circ a \circ a \circ z$. Thus

$$
a \leq a \circ x \circ a \leq a \circ x \circ a \circ x \circ a=a \circ x \circ(y \circ a \circ a \circ z) \circ x \circ a=(a \circ x \circ y \circ a) \circ(a \circ z \circ x \circ a) .
$$

Then for some $p \in a \circ x \circ y \circ a$ and $q \in a \circ z \circ x \circ a$ we have $a \leq p \circ q$ that is $(p, q) \in A_{a}$. Since $A_{a} \neq \emptyset$, we have

$$
\left(f_{B} \widetilde{*} f_{B}\right)(a)=\bigcap_{\left(p_{1}, q_{1}\right) \in A_{a}}\left\{f_{B}\left(p_{1}\right) \cup f_{B}\left(q_{1}\right)\right\} \subseteq\left\{f_{B}(p) \cup f_{B}(q)\right\}
$$

As $f_{B}$ is a uni-soft bi-hyperideal of $S$ over $U$, we have

$$
\bigcup_{\substack{p \in a \circ \delta \circ a \\ \delta \in x \circ y}} f_{B}(p) \subseteq\left\{f_{B}(a) \cup f_{B}(a)\right\}=f_{B}(a) .
$$

and

$$
\bigcup_{\substack{q \in a \circ \gamma \circ a \\ \gamma \in z \circ x}} f_{B}(q) \subseteq\left\{f_{B}(a) \cup f_{B}(a)\right\}=f_{B}(a) .
$$

Thus

$$
\left(f_{B} \widetilde{*} f_{B}\right)(a) \subseteq\left\{f_{B}(p) \cup f_{B}(q)\right\} \subseteq\left\{f_{B}(a) \cup f_{B}(a)\right\}=f_{B}(a) .
$$

Hence, $f_{B} \widetilde{\supseteq} f_{B} \widetilde{*} f_{B}$ by Proposition 3.10 , we have $f_{B} \widetilde{*} f_{B} \widetilde{\supseteq} f_{B}$. Therefore, $f_{B} \widetilde{*} f_{B}=f_{B}$.

$(2) \Leftrightarrow(3)$. Let $g_{C}$ and $h_{D}$ be uni-soft bi-hyperideals of $S$ over $U$. Then $g_{C} \widetilde{\cup} h_{D}$ is a uni-soft bi-hyperideal of $S$ over $U$. By (2),

$$
g_{C} \widetilde{\cup} h_{D}=\left(g_{C} \widetilde{\cup} h_{D}\right) \widetilde{*}\left(g_{C} \widetilde{\cup} h_{D}\right) \widetilde{\supseteq} g_{C} \widetilde{*} h_{D} .
$$

Similarly, we can prove that $g_{C} \widetilde{\cup} h_{D} \widetilde{\supseteq} h_{D} \widetilde{*} g_{C}$. Thus $g_{C} \widetilde{\cup} h_{D} \widetilde{\supseteq}\left(g_{C} \widetilde{*} h_{D}\right) \widetilde{\cup}\left(h_{D} \widetilde{*} g_{C}\right)$. For the reverse inclusion, by Lemma 3.13, $g_{C} \widetilde{*} h_{D}$ and $h_{D} \widetilde{*} g_{C}$ are uni-soft bi-hyperideals of $S$ over $U$. So $\left(g_{C} \widetilde{*} h_{D}\right) \widetilde{\cup}\left(h_{D} \widetilde{*} g_{C}\right)$ is a uni-soft bi-hyperideal of $S$ over $U$. By $(2)$ we have

$$
\begin{aligned}
& \left(g_{C} \widetilde{*} h_{D}\right) \widetilde{\cup}\left(h_{D} \widetilde{*} g_{C}\right)=\left(\left(g_{C} \widetilde{*} h_{D}\right) \widetilde{\cup}\left(h_{D} \widetilde{*} g_{C}\right)\right) \widetilde{*}\left(\left(g_{C} \widetilde{*} h_{D}\right) \widetilde{\cup}\left(h_{D} \widetilde{*} g_{C}\right)\right) \\
& \widetilde{\supseteq} g_{C} \widetilde{*} h_{D} \widetilde{*} h_{D} \widetilde{*} g_{C}=g_{C} \widetilde{*}\left(h_{D} \widetilde{*} h_{D}\right) \widetilde{*} g_{C} \\
& =g_{C} \widetilde{*} h_{D} \widetilde{*} g_{C}\left(\text { as } h_{D} \widetilde{*} h_{D}=h_{D} \text { by }(2)\right) \\
& \widetilde{\supseteq} g_{C} \widetilde{*} \mathcal{S}_{\mathcal{S}}^{c} \widetilde{*} g_{C}\left(\text { as } g_{C} \widetilde{*} h_{D} \widetilde{\supseteq} g_{C} \widetilde{*} \mathcal{S}_{\mathcal{S}}^{c}\right. \text { by Lemma 3.6. } \\
& \widetilde{\supseteq} g_{C}\left(\text { as } g_{C} \widetilde{*} \mathcal{S}_{\mathcal{S}}^{c} \widetilde{*} g_{C} \supseteq g_{C}\right. \text { by Proposition 3.10). }
\end{aligned}
$$


Hence, $\left(g_{C} \widetilde{*} h_{D}\right) \widetilde{\cup}\left(h_{D} \widetilde{*} g_{C}\right) \supseteq g_{C}$. Similarly we can prove that $\left(g_{C} \widetilde{*} h_{D}\right) \widetilde{\cup}\left(h_{D} \widetilde{*} g_{C}\right) \supseteq h_{D}$. Thus $\left(g_{C} \widetilde{*} h_{D}\right) \widetilde{\cup}\left(h_{D} \widetilde{*} g_{C}\right) \simeq g_{C} \widetilde{\cup} h_{D}$. Therefore, $\left(g_{C} \widetilde{*} h_{D}\right) \widetilde{\cup}\left(h_{D} \widetilde{*} g_{C}\right)=g_{C} \widetilde{\cup} h_{D}$.

$(3) \Leftrightarrow(1)$. Let $g_{C}$ be a uni-soft right hyperideal and $h_{D}$ be a uni-soft left hyperideal of $S$ over $U$, then $g_{C}$ and $h_{D}$ are uni-soft bi-hyperideals of $S$ over $U$. Hence, by hypothesis, $\left(g_{C} \widetilde{*} h_{D}\right) \widetilde{\cup}\left(h_{D} \widetilde{*} g_{C}\right)=$ $g_{C} \widetilde{\cup} h_{D}$. Thus by Theorem 4.7, $S$ is both regular and intra-regular.

$(3) \Rightarrow(4)$. Let $f_{A}$ and $g_{B}$ be uni-soft bi-hyperideals of $S$ over $U$ such that $f_{A} \widetilde{*} f_{A} \widetilde{\supseteq} g_{B}$. By hypothesis

$$
f_{A}=f_{A} \widetilde{\cup} f_{A}=\left(f_{A} \widetilde{*} f_{A}\right) \widetilde{\cup}\left(f_{A} \widetilde{*} f_{A}\right)=f_{A} \widetilde{*} f_{A} .
$$

Thus $f_{A} \widetilde{\supseteq} g_{B}$. Hence, every uni-soft bi-hyperideal of $S$ over $U$ is semiprime.

$(4) \Rightarrow(5)$. Let $f_{A}$ be a proper uni-soft bi-hyperideal of $S$ over $U$ and $\left\{f_{A_{i}}: i \in I\right\}$ be the collection of all irreducible uni-soft bi-hyperideals of $S$ over $U$ which contained in $f_{A}$. By Proposition 4.6, this collection is nonempty. Hence, $f_{A} \widetilde{\supseteq} \bigcup_{i \in I} f_{A_{i}}$. Let $a \in S$, then by Proposition 4.6, there exists an irreducible uni-soft bi-hyperideal $f_{A_{\alpha}}$ of $S$ over $U$ such that $f_{A} \cong f_{A_{\alpha}}$ and $f_{A}(a)=f_{A_{\alpha}}(a)$. Thus $f_{A_{\alpha}} \in\left\{f_{A_{i}}: i \in I\right\}$. Hence, $\bigcup_{i \in I} f_{A_{i}} \cong f_{A_{\alpha}}$. So $\bigcup_{i \in I} f_{A_{i}}(a) \supseteq f_{A_{\alpha}}(a)=f_{A}(a)$. Thus $\bigcup_{i \in I} f_{A_{i}} \supseteq f_{A}$. Consequently $\bigcup_{i \in I} f_{A_{i}}=f_{A}$. By hypothesis each uni-soft bi-hyperideal of $S$ over $U$ is semiprime. So each uni-soft bi-hyperideal of $S$ over $U$ is the union of all irreducible semiprime uni-soft bi-hyperideals of $S$ over $U$ which contained in.

$(5) \Rightarrow(2)$. Let $f_{A}$ be a uni-soft bi-hyperideal of $S$ over $U$. Then by Lemma 3.13, $f_{A} \widetilde{*} f_{A}$ is also uni-soft bi-hyperideal of $S$ over $U$. Since $f_{A}$ is a uni-soft semihypergroup of $S$ over $U$ so by Proposition 3.12, $f_{A} \widetilde{*} f_{A} \widetilde{\supseteq} f_{A}$. By hypothesis $f_{A} \widetilde{*} f_{A}=\bigcup_{i \in I} f_{A_{i}}$ where $f_{A_{i}}$ are irreducible semiprime uni-soft bi-hyperideals of $S$ over $U$. Thus $f_{A} \widetilde{*} f_{A} \widetilde{\supseteq} f_{A_{i}}$ for all $i \in I$. Hence, $f_{A} \widetilde{\supseteq} f_{A_{i}}$ for all $i \in I$, because each $f_{A_{i}}$ is semiprime. Thus $f_{A} \widetilde{\supseteq} \bigcup_{i \in I} f_{A_{i}}=f_{A} \widetilde{*} f_{A}$. Hence, $f_{A} \widetilde{*} f_{A}=f_{A}$.

Proposition 4.9. Let $S$ be both regular and intra-regular ordered semihypergroup. Then the following statements are equivalent:

(1) Every uni-soft bi-hyperideal of $S$ over $U$ is strongly irreducible.

(2) Every uni-soft bi-hyperideal of $S$ over $U$ is strongly prime.

Proof. $(1) \Rightarrow(2)$. Let $S$ be both regular and intra-regular ordered semihypergroup and $f_{A}$ be a strongly irreducible uni-soft bi-hyperideal of $S$ over $U$. Let $g_{B}$ and $h_{C}$ be uni-soft bi-hyperideals of $S$ over $U$ such that $\left(g_{B} \widetilde{*} h_{C}\right) \widetilde{\cup}\left(h_{C} \widetilde{*} g_{B}\right) \widetilde{\supseteq} f_{A}$. Since $S$ is both regular and intra-regular, by Theorem 4.8, $\left(g_{B} \widetilde{*} h_{C}\right) \widetilde{\cup}\left(h_{C} \widetilde{*} g_{B}\right)=g_{B} \widetilde{\cup} h_{C}$. Thus $g_{B} \widetilde{\cup} h_{C} \supseteq f_{A}$. Since $f_{A}$ is a strongly irreducible, we have either $g_{B} \supseteq f_{A}$ or $h_{C} \supseteq f_{A}$. Thus $f_{A}$ is a strongly prime uni-soft bi-hyperideal of $S$ over $U$.

$(2) \Rightarrow(1)$. Suppose $f_{A}$ is a strongly prime uni-soft bi-hyperideal of $S$ over $U$ and $g_{B}$ and $h_{C}$ be uni-soft bi-hyperideals of $S$ over $U$ such that $g_{B} \widetilde{\cup} h_{C} \widetilde{\supseteq} f_{A}$. As $\left(g_{B} \widetilde{*} h_{C}\right) \widetilde{\cup}\left(h_{C} \widetilde{*} g_{B}\right) \widetilde{\supseteq} g_{B} \widetilde{\cup} h_{C} \widetilde{\supseteq} f_{A}$. Since $f_{A}$ is strongly prime, we get either $g_{B} \widetilde{\supseteq} f_{A}$ or $h_{C} \widetilde{\supseteq} f_{A}$. Thus $f_{A}$ is a strongly irreducible uni-soft bi-hyperideal of $S$ over $U$.

Theorem 4.10. Each uni-soft bi-hyperideal of an ordered semihypergroup $S$ is strongly prime if and only if $S$ is both regular and intra-regular and the set of uni-soft bi-hyperideals of $S$ over $U$ is totally ordered under inclusion. 
Proof. Suppose that each uni-soft bi-hyperideal of $S$ over $U$ is strongly prime. Then each uni-soft bi-hyperideal of $S$ over $U$ is semiprime. Thus by Theorem 4.8, $S$ is both regular and intraregular. To prove that the set of uni-soft bi-hyperideals of $S$ over $U$ is totally ordered under inclusion. Let $g_{B}$ and $h_{C}$ be any two uni-soft bi-hyperideals of $S$ over $U$. Then by Theorem 4.8, $\left(g_{B} \widetilde{*} h_{C}\right) \widetilde{\cup}\left(h_{C} \widetilde{*} g_{B}\right)=g_{B} \widetilde{\cup} h_{C}$. As each uni-soft bi-hyperideal of $S$ is strongly prime, we obtain $g_{B} \widetilde{\cup} h_{C}$ is strongly prime. Hence, either $g_{B} \widetilde{\supseteq} g_{B} \widetilde{\cup} h_{C}$ or $h_{C} \widetilde{\supseteq} g_{B} \widetilde{\cup} h_{C}$. If $g_{B} \widetilde{\supseteq} g_{B} \widetilde{\cup} h_{C}$, then $g_{B} \widetilde{\supseteq} h_{C}$ and if $h_{C} \widetilde{\supseteq} g_{B} \widetilde{\cup} h_{C}$, then $h_{C} \widetilde{\supseteq} g_{B}$.

Conversely, assume that $S$ is both regular and intra-regular and the set of uni-soft bi-hyperideals of $S$ over $U$ is totally ordered under inclusion. Then each uni-soft bi-hyperideal of $S$ over $U$ is strongly prime. Indeed: Let $f_{A}$ be an arbitrary uni-soft bi-hyperideal of $S$ over $U$ and $g_{B}, h_{C}$ be any uni-soft bi-hyperideals of $S$ over $U$ such that $\left(g_{B} \widetilde{*} h_{C}\right) \widetilde{\cup}\left(h_{C} \widetilde{*} g_{B}\right) \simeq f_{A}$. Since $S$ is both regular and intra-regular, by Theorem 4.8, $\left(g_{B} \widetilde{*} h_{C}\right) \widetilde{\cup}\left(h_{C} \widetilde{*} g_{B}\right)=g_{B} \widetilde{\cup} h_{C}$. Thus $g_{B} \widetilde{\cup} h_{C} \widetilde{\supseteq} f_{A}$. Since the set of uni-soft bi-hyperideals of $S$ over $U$ is totally ordered, we have either $g_{B} \widetilde{\supseteq} h_{C}$ or $h_{C} \widetilde{\supseteq} g_{B}$ that is either $g_{B} \widetilde{\cup} h_{C}=g_{B}$ or $g_{B} \widetilde{\cup} h_{C}=h_{C}$. Thus either $g_{B} \supseteq f_{A}$ or $h_{C} \supseteq f_{A}$. Hence, $f_{A}$ is a strongly prime uni-soft bi-hyperideal of $S$ over $U$.

Theorem 4.11. If the set of uni-soft bi-hyperideals of an ordered semihypergroup $S$ over $U$ is totally ordered under inclusion, then $S$ is both regular and intra-regular if and only if each uni-soft bi-hyperideal of $S$ over $U$ is prime.

Proof. Suppose $S$ is both regular and intra-regular. Let $f_{B}$ be any uni-soft bi-hyperideal of $S$ over $U$, and $g_{C}, h_{D}$ are uni-soft bi-hyperideals of $S$ over $U$ such that $g_{C} \widetilde{*} h_{D} \widetilde{\supseteq} f_{B}$. Since the set of uni-soft bi-hyperideals of $S$ over $U$ is totally ordered under inclusion, we get that either $g_{C} \supseteq h_{D}$ or $h_{D} \supseteq g_{C}$. Suppose $g_{C} \supseteq h_{D}$, then $g_{C} \widetilde{*} g_{C} \widetilde{\supseteq} g_{C} \widetilde{*} h_{D} \supseteq f_{B}$. By Theorem 4.8, $f_{B}$ is semiprime, so $g_{C} \widetilde{\supseteq} f_{B}$. Hence, $f_{B}$ is a prime uni-soft bi-hyperideals of $S$ over $U$.

Conversely, assume that every uni-soft bi-hyperideal of $S$ over $U$ is prime. Since every prime uni-soft bi-hyperideal is semiprime, so by Theorem $4.8, S$ is both regular and intra-regular.

Theorem 4.12. Let $S$ be an ordered semihypergroup. Then the following assertions are equivalents:

(1) The set of uni-soft bi-hyperideals of $S$ over $U$ is totally ordered under inclusion.

(2) Each uni-soft bi-hyperideal of $S$ over $U$ is strongly irreducible.

(3) Each uni-soft bi-hyperideal of $S$ over $U$ is irreducible.

Proof. $(1) \Rightarrow(2)$. Let $f_{B}, g_{C}$ and $h_{D}$ be uni-soft bi-hyperideals of $S$ over $U$ such that $g_{C} \widetilde{\cup} h_{D} \widetilde{\precsim} f_{B}$. Since the set of uni-soft bi-hyperideals of $S$ over $U$ is totally ordered, we get that either $g_{C} \supseteq h_{D}$ or $h_{D} \widetilde{\supseteq} g_{C}$. Thus either $g_{C} \widetilde{\cup} h_{D}=g_{C}$ or $g_{C} \widetilde{\cup} h_{D}=h_{D}$. Hence, $g_{C} \widetilde{\cup} h_{D} \widetilde{\supseteq} f_{B}$ implies either $g_{C} \widetilde{\supseteq} f_{B}$ or $h_{D} \widetilde{\supseteq} f_{B}$. Thus $f_{B}$ is strongly irreducible.

$(2) \Rightarrow(3)$. Let $f_{B}$ be an arbitrary uni-soft bi-hyperideal of $S$ over $U$ and $g_{C}, h_{D}$ be two unisoft bi-hyperideals of $S$ over $U$ such that $g_{C} \widetilde{\cup} h_{D}=f_{B}$. Then $f_{B} \widetilde{\supseteq} g_{C}$ and $f_{B} \widetilde{\supseteq} h_{D}$. By hypothesis either $g_{C} \widetilde{\supseteq} f_{B}$ or $h_{D} \supseteq f_{B}$. So either $g_{C}=f_{B}$ or $h_{D}=f_{B}$. That is $f_{B}$ is an irreducible uni-soft bi-hyperideal of $S$ over $U$.

$(3) \Rightarrow(1)$. Let $g_{C}, h_{D}$ be any two uni-soft bi-hyperideals of $S$ over $U$. Then $g_{C} \widetilde{\cup} h_{D}$ is a unisoft bi-hyperideal of $S$ over $U$. Also $g_{C} \widetilde{\cup} h_{D}=g_{C} \widetilde{\cup} h_{D}$. So by hypothesis either $g_{C} \widetilde{\cup} h_{D}=g_{C}$ or $g_{C} \widetilde{\cup} h_{D}=h_{D}$, that is either $g_{C} \supseteq h_{D}$ or $h_{D} \widetilde{\supseteq} g_{C}$. Hence, the set of all uni-soft bi-hyperideals of $S$ over $U$ is totally ordered under inclusion. 


\section{Conclusion}

In this paper, we have applied uni-soft set theory in hyperstructure, in particular, in ordered semihypergroups. We introduced the notions of uni-soft bi-hyperideals in ordered semihypergroups. Moreover we introduced the notions of prime, (strongly prime, semiprime, irreducible, and strongly irreducible) uni-soft bi-hyperideals of ordered semihypergroups. We considered characterizations of different classes in terms of these newly defined uni-soft hyperideals. Seems that the obtained characterizations will be very useful for future study of ordered semihypergroups and their applications. In our future study of soft hyperstructures, we will apply the above new idea to other algebraic structures.

\section{References}

[1] H. Aktas, N. Cağman, Soft sets and soft groups, Information Sciences, 177 (2007), 2726-2735.

[2] M.I. Ali, A note on soft sets, rough sets and fuzzy sets, Applied Soft Computing, 11 (2011), 3329-3332.

[3] M.I. Ali, F. Feng, X.Y. Liu, W.K. Min, M. Shabir, On some new operations in soft set theory, Computers and Mathematics with Applications, 57 (2009), 1547-1553.

[4] M.I. Ali, M. Shabir, M. Naz, Algebraic structures of soft sets associated with new operations, Computers and Mathematics with Applications, 61 (2011), 2647-2654.

[5] T. Changphas, B. Davvaz, Bi-hyperideals and quasi-hyperideals in ordered semihypergroups, Italian Journal of Pure and Applied Mathematics, 35 (2015), 493-508.

[6] P. Corsini, V. Leoreanu-Fotea, Applications of hypersturcture theory, Advanced in Mathematics, Kluwer Academic Publisher, 2003.

[7] B. Davvaz, A. Khan, M. Farooq, Int-soft structures applied to ordered semihypergroups, Le Matematiche, 73 (2018), 235-259.

[8] F. Feng, Y.B. Jun, X. Zhao, Soft semirings, Computers and Mathematics with Applications, 56 (2008), 2621-2628.

[9] F. Feng, Y.M. Li, Soft subsets and soft product operations, Information Sciences, 232 (2013), $44-57$.

[10] F. Feng, C. Li, B. Davvaz, M.I. Ali, Soft sets combined with fuzzy sets and rough sets: A tentative approach, Soft Computing, 14 (2010), 899-911.

[11] M. Farooq, A. Khan, B. Davvaz, Characterizations of ordered semihypergroups by the properties of their intersectional-soft generalized bi-hyperideals, Soft Computing, 22 (2018), 30013010 .

[12] M. Farooq, A. Khan, M. Izhar, B. Davvaz, $(\mathcal{M}, \mathcal{N})$-Int-soft generalized bi-hyperideals of ordered semihypergroups, Journal of New Theory, 23 (2018), 31-47.

[13] M. Farooq, T. Mahmood, A. Khan, M. Izhar, B. Davvaz, Fuzzy hyperideals of hyperquantales, Journal of Intelligent and Fuzzy Systems, 36 (2019), 5605-5615. 
[14] D. Heidari, B. Davvaz, On ordered hyperstructures, Politehn. UPB Scientific Bulletin, Series A: Applied Mathematics and Physics, 73(2) (2011), 85-96.

[15] Y.B. Jun, Y.J. Lee, A. Khan, Soft ordered semigroups, Mathematical Logic Quarterly, 56 (2010), 42-50.

[16] Y.B. Jun, S.Z. Song, G. Muhiuddin, Concave soft sets, critical soft points, and union-soft ideals of ordered semigroups, The Scientific World Journal, 2014 (2014), 1-11.

[17] A. Khan, M. Farooq, H.U. Khan, Uni-soft hyperideals of ordered semihypergroups, Journal of Intelligent and Fuzzy Systems, 35 (2018), 4557-4571.

[18] A. Khan, M. Farooq, N. Yaqoob, Uni-soft structures applied to ordered $\Gamma$-semihypergroups, Proceedings of the National Academy of Sciences, India Section A: Physical Sciences, (2019), https://doi.org/10.1007/s40010-019-00602-x.

[19] A. Khan, Y.B. Jun, S.I.A. Shah, R. Khan, Applications of soft union sets in ordered semigroups via uni-soft quasi-ideals, Journal of Intelligent and Fuzzy Systems, 30 (2016), 97-107.

[20] A. Khan, R. Khan, Y.B. Jun, Uni-soft structure applied to ordered semigroups, Soft Computing, 21 (2017), 1021-1030.

[21] P.K. Maji, R. Biswas, R. Roy, Soft set theory, Computers and Mathematics with Applications, 45 (2003), 555-562.

[22] P. Majumdar, S.K. Samanta, Generalized fuzzy soft sets, Computers and Mathematics with Applications, 59 (2010), 1425-1432.

[23] F. Marty, Sur Une generalization de la notion de group, $8^{\text {iem }}$ Congress, Math Scandinaves Stockholm, (1934), 45-49.

[24] D. Molodtsov, Soft set theory-first results, Computers and Mathematics with Applications, 37 (1999), 19-31.

[25] S. Naz, M. Shabir, On prime soft bi-hyperideals of semihypergroups, Journal of Intelligent and Fuzzy Systems, 26 (2014), 1539-1546.

[26] S. Omidi, B. Davvaz, J. Zhan, An investigation on ordered algebraic hyperstructures, Acta Mathematica Sinica, English Series, 33(8) (2017), 1107-1124.

[27] B. Pibaljommee, B. Davvaz, Characterizations of (fuzzy) bi-hyperideals in ordered semihypergroups, Journal of Intelligent and Fuzzy Systems, 28 (2015), 2141-2148.

[28] W. Xu, J. Ma, S. Wang, G. Hao, Vague soft sets and their properties, Computers and Mathematics with Applications, 59 (2010), 787-794.

[29] J. Zhan, Q. Liu, B. Davvaz, A new rough set theory: Rough soft hemirings, Journal of Intelligent and Fuzzy Systems, 28 (2015), 1687-1697. 